\title{
Landau Theory of the Reentrant Nematic-Smectic A Phase Transition
}

\section{Citation}

Pershan, Peter S., and J. Prost. 1979. Landau theory of the reentrant nematic-smectic A phase transition. Journal de Physique 40(2): 27-30.

\section{Published Version}

doi:10.1051/jphyslet:0197900400202700

\section{Permanent link}

http://nrs.harvard.edu/urn-3:HUL.InstRepos:10361975

\section{Terms of Use}

This article was downloaded from Harvard University's DASH repository, and is made available under the terms and conditions applicable to Other Posted Material, as set forth at http:// nrs.harvard.edu/urn-3:HUL.InstRepos:dash.current.terms-of-use\#LAA

\section{Share Your Story}

The Harvard community has made this article openly available.

Please share how this access benefits you. Submit a story.

Accessibility 


\title{
Landau theory of the reentrant nematic-smectic A phase transition
}

\author{
P. S. Pershan $\left(^{*}\right)$ \\ Division of Applied Sciences, Harvard University, Cambridge, Massachusetts 02138, U.S.A. \\ and J. Prost \\ Centre Paul-Pascal, CNRS, Domaine Universitaire, 33405 Talence, France.
}

(Reçu le 2 octobre 1978, révisé le 28 novembre 1978, accepté le 29 novembre 1978)

\begin{abstract}
Résumé. - Nous montrons que le diagramme de phase rentrant de la transition nématique-smectique A, découle naturellement de la théorie de Landau si l'on admet qu'il existe une densité optimale pour l'obtention de l'ordre smectique. La forme de la ligne de coexistence dans le plan $P-T$, est correctement prédite par la théorie. Nous décrivons aussi le comportement rentrant des mélanges.
\end{abstract}

\begin{abstract}
The reentrant, nematic to smectic A phase transition is shown to follow from the Landau theory if one assumes the existence of an optimum density for smectic ordering. The shape of the coexistence line in the $P-T$ plane is fit exactly by this theory. The effects of concentration on reentrant behaviour are also explained.
\end{abstract}

Cladis and coworkers have recently demonstrated that for some liquid crystals, the boundary between nematic and smectic A phases in the $P, T$ plane is reentrant in that at high enough pressures the nematic phase exists for temperatures both lower and higher than for the smectic phase [1]. In some materials that have a smectic-A to nematic phase transition which is not reentrant, reentrant behaviour can be induced by mixing it with a second liquid crystal that only has a nematic phase [2]. Cladis also observed that reentrant behaviour appears only in materials for which the smectic phase is of the bilayer type and suggested that at densities above some optimum value the interactions stabilizing smectic order would be suppressed by steric effects. The purpose of this article is to demonstrate that if one assumes the existence of this optimum density most of the observed properties of the reentrant transition are predicted by the Landau theory of the nematic to smectic A phase transition $[3,4]$.

Reentrant behaviour has also been observed at the normal to superconducting phase boundary in materials containing magnetic impurities [5] and in one of the ${ }^{3} \mathrm{He}$ phase transitions [6]. In view of the often used analogy between smectics and superfluids [3, 4] it is important to note that reentrant behaviour is simply a consequence of two competing interactions

(*) This work was supported in part by the National Science Foundation under Grant No. NSF DMR 76-22452. whose sum can be optimized through control of another variable. Its appearance in superconductors, ${ }^{3} \mathrm{He}$ and liquid crystals does not necessarily imply the microscopic interactions responsible for the effects, are analogous.

In the usual form of the Landau theory the difference between the free energy per unit mass of the smectic and nematic phases is expanded as a power series in the smectic order parameter $|\psi|$

$$
F_{\mathrm{s}}-F_{\mathrm{N}}=f=\frac{1}{2} A|\psi|^{2}+\frac{1}{4} B|\psi|^{4}+\frac{1}{6} C|\psi|^{6}
$$

where $A \equiv a\left(T-T^{*}\right)$. If $B>0$ the transition is second order and occurs at $T=T^{*}$; if $B<0$ a first order transition occurs at $T=T^{*}+3 B^{2} / 16 a C$.

The Landau theory has been widely applied to a number of different phase transitions and examples where the order parameter couples to some other variable are also common $[4,7]$. In the present case we want to describe coupling of $|\psi|$ to both the density $\rho$ and the relative concentration $x$ of a binary mixture. The simplest assumption is to add a term to eq. (1) of the form $g(\rho, x)|\psi|^{2}$, expand $g$ as a power series in $\left(\rho-\rho_{0}, x-x_{0}\right)$ and keep only the leading terms. If we consider only the density term first, and choose $\rho_{0}$ to be the optimum density referred to above, the $T^{*}$ appearing in eq. (1) can be replaced by

$$
T^{*}(x, \rho)=T_{0}(x)+t_{2}\left(\rho-\rho_{0}\right)^{2}
$$


where $t_{2}<0$ insures that $\rho \neq \rho_{0}$ suppresses the phase transition.

The general procedure [8] for obtaining the nematic to smectic phase boundary is to first obtain expressions for the pressure $P=\rho^{2}(\partial F / \partial \rho)_{x, T}$, and the chemical potentials

$$
\mu^{\prime}=(\partial F / \partial x)_{\rho, T}
$$

and

$$
\mu=F-x(\partial F / \partial x)_{\rho, T}+\rho(\partial F / \partial \rho)_{x, T}
$$

for the two components and for both phases. Setting $\mu, \mu^{\prime}$, and $P$ for the smectic phase equal to those of the nematic phase defines the phase boundary. From the equality of $P$ one obtains the difference $\rho^{\mathbf{s}}-\rho^{N}$ between the smectic and nematic densities

$$
\rho^{\mathrm{s}}-\rho^{\mathrm{N}} \simeq \frac{a}{2} \rho^{2}\left(\frac{\partial T^{*}}{\partial \rho}\right)_{x}|\psi|^{2} \quad \frac{\partial}{\partial \rho}(P)_{x}
$$

where the derivatives are evaluated at $\rho=\rho^{\mathrm{N}}$ and $x=x^{\mathrm{N}}$. From the equality of $\mu^{\prime}$ one obtains

$$
x^{\mathbf{s}}-x^{\mathrm{N}} \simeq \frac{a}{2}\left(\frac{\partial T^{*}}{\partial x}\right)_{\rho}|\psi|^{2},\left(\frac{\partial \mu^{\prime}}{\partial x}\right)_{\rho}
$$

and the condition that $\mu_{\mathrm{s}}-\mu_{\mathrm{N}}=0$ is only slightly different from the usual condition that $F_{\mathrm{s}}-F_{\mathrm{N}}=0$ at the transition. It has exactly the same form, however, $T^{*}(x, \rho)$ is replaced by $T^{*}\left(x^{\mathbf{N}}, \rho^{\mathrm{N}}\right)$ and $B$ is changed by the addition of two negative terms

$$
-\frac{a^{2}}{2}\left[\frac{\left(\partial T^{*} / \partial x\right)_{\rho}^{2}}{\left(\frac{\partial \mu^{\prime}}{\partial x}\right)_{\rho}}+\frac{\left(\partial T^{*} / \partial \rho\right)_{x}^{2}}{\frac{\partial}{\partial \rho}\left(\frac{P}{\rho^{2}}\right)_{x}}\right] .
$$

The equation obtained by minimizing $\mu_{\mathrm{s}}-\mu_{\mathrm{N}}$ with respect to $\psi$ for given $\rho^{\mathbf{N}}$ and $x^{\mathrm{N}}$ is identical to the equation and the result obtained by minimizing eq. (1) at fixed $\rho^{\mathrm{s}}$ and $x^{\mathrm{s}}$ if the equalities described by eqs. (3) and (4) are taken into account. If the terms described by eq. (5) are small in comparison with the original value of $|B|$ they can be neglected regardless of whether $B<0$, implying a first order transition, or $B>0$ implying one of second order. On the other hand, since coupling between $|\psi|^{2}$ and either $\rho$ or $x$ makes a negative contribution to $B$ it also increases the tendency to first order behaviour. Also, since for dilute solutions $(\partial \mu / \partial x)^{-1} \propto x$ one can reasonably expect that the effect of adding a second component to a pure material that exhibits a second order $\mathrm{s}-\mathrm{N}$ transition may be to induce a tricritical point at some finite $x$.

Neglecting the terms described by eq. (5) the transition temperature can be obtained from eqs. (1) and (2) by setting $T-T^{*}\left(x^{\mathbf{N}}, \rho^{\mathbf{N}}\right)$ equal to either zero for a second order transition or $3 B^{2} / 16 a C$ if it is first order. Taking

$$
\left(\rho^{\mathrm{N}}-\rho_{0}\right) / \rho_{0}=-\alpha_{\mathrm{N}}\left(T-T_{0}\right)+\beta_{\mathrm{N}}\left(P-P_{0}\right),
$$

where $\alpha_{\mathrm{N}}$ and $\beta_{\mathrm{N}}$ are respectively the thermal expansion and compressibility for the nematic phase. The phase boundary surface is predicted to have the form

$$
T_{3}\left[T_{1}(x)-T\right]=\left(T_{2}-T+\left[\beta_{\mathrm{N}} / \alpha_{\mathrm{N}}\right] P\right)^{2} .
$$

In figure 1 we show the fit of eq. (6) to data reported by Cladis et al. for a sample of pure 4-cyano-4'octyloxy biphenyl (8OCB) [1]. In terms of the parameters of eq. (6) the maximum temperature, is indicated in figure 1 by $T_{\text {(II) }}=T_{1}(x)$ where $x=0$, the pressure for this temperature $P_{(\mathrm{II})}=\left(\alpha_{\mathrm{N}} / \beta_{\mathrm{N}}\right)\left(T_{\mathrm{II}}-T_{2}\right)$, the temperature, for which the pressure is a maximum $T_{\mathrm{I}}=T_{\mathrm{II}}-T_{3} / 4$ and $P_{\mathrm{I}}=\left(\alpha_{\mathrm{N}} / \beta_{\mathrm{N}}\right)\left[T_{\mathrm{II}}+T_{3} / 4-T_{2}\right]$. The parameters for this fit are

$$
T_{1}=83^{\circ} \mathrm{C}, \quad T_{2}=46.2^{\circ} \mathrm{C}, \quad T_{3}=22^{\circ} \mathrm{C}
$$

and

$$
\beta_{\mathrm{N}} / \alpha_{\mathrm{N}}=23 \mathrm{~K} / \mathrm{kbar} \text {. }
$$

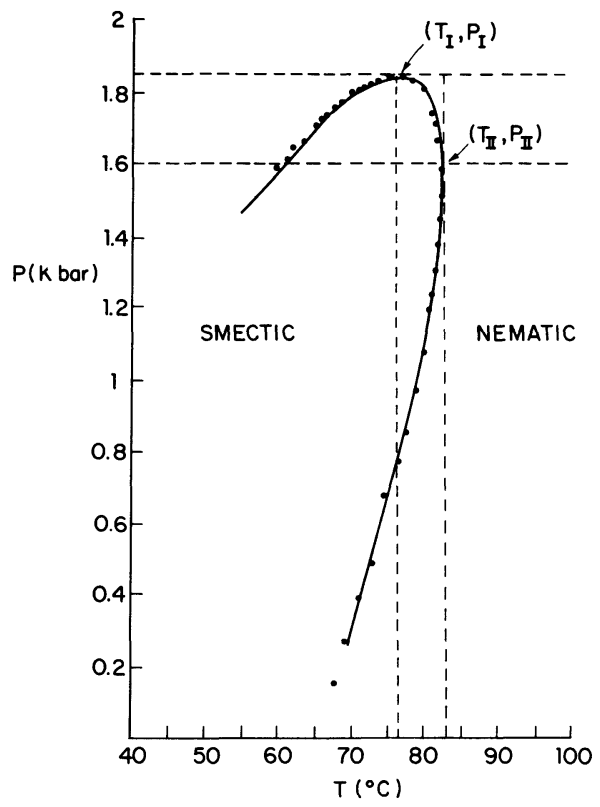

Fig. 1. - Typical reentrant nematic phase diagram. The dots are the experimentally determined points for $80 C B$ (see Ref. [1]). The solid line results from eq. (6) with $x=0$ and parameters as specified in the text.

Although $T_{1}, T_{2}$ and $T_{3}$ are completely adjustable $\beta_{\mathrm{N}} / \alpha_{\mathrm{N}}$ can be estimated since the thermal expansion coefficient $\alpha_{\mathrm{N}} \sim 10^{-3} \mathrm{~K}^{-1}$ for almost all condensed fluids and also for nematic liquid crystals [9, 10]. Also $\beta_{\mathbf{N}}$ can be obtained from sound speeds in either the isotropic or nematic phases [11]. Typical values for similar materials obtain $\beta_{\mathrm{N}}^{-1} \sim 4$ or $5 \times 10^{10}$ dyne. $\mathrm{cm}^{-2} \simeq 40$ or $50 \mathrm{kbar}$ obtaining very good agreement between the expected value of $\beta_{\mathrm{N}} / \alpha_{\mathrm{N}} \sim 20$ to $25 \mathrm{~K} / \mathrm{kbar}$, and the value that fits the data. This is the strongest evidence sup- 
porting the premise that the phase boundary curvature can be interpreted in terms of an optimum density for smectic order.

Cladis already noted that in binary mixtures of N-p-cyanobenzylidene-p-nonylaniline (CBNA) and $\mathrm{N}$-p-cyanobenzylidene-p-heptylaniline (CBHA) the maximum pressure $P_{\mathrm{I}}$ is a linear function of the ratio of CBNA to CBHA [12]. This follows from the above considerations if we take $T_{0}(x)$ to be a linear function of this ratio, e.g.,

$$
T_{0}(x)=T_{0}+t_{1} y=T_{0}+t_{1}[x /(1-x)]
$$

where

$$
y=[\mathrm{CBNA}] /[\mathrm{CBHA}]=x /(1-x) .
$$

Since $T_{1}(x)-T_{0}(x)$ is a constant $P_{\mathrm{I}}$ is also linear in $x /(1-x)$. Figure 2 contains one possible fit of eq. (6) to Cladis's data for this mixture using

$$
\begin{gathered}
T_{1}=42.85^{\circ} \mathrm{C}, \quad T_{2}=43.55^{\circ} \mathrm{C}, \quad T_{3}=25 \mathrm{~K}, \\
t_{1}=38.7 \mathrm{~K} \text { and } \beta_{\mathrm{N}} / \alpha_{\mathrm{N}}=19.4 \mathrm{~K} / \mathrm{kbar}
\end{gathered}
$$

Although the linear dependence of $T^{*}$ on $x(1-x)^{-1}$ is ad hoc it is interesting that it is sufficient to qualitatively describe all of the concentration effects. Furthermore, the ratio $\alpha_{N} / \beta_{N}$ is in reasonable agreement with the values expected from an optimum density model. More generally, one would certainly expect $\rho_{0}$ to depend on $x$ and there is no reason why the other parameters shouldn't also. Although inclusion of these effects would undoubtably improve the fit it does not seem worthwhile without some specific microscopic model.

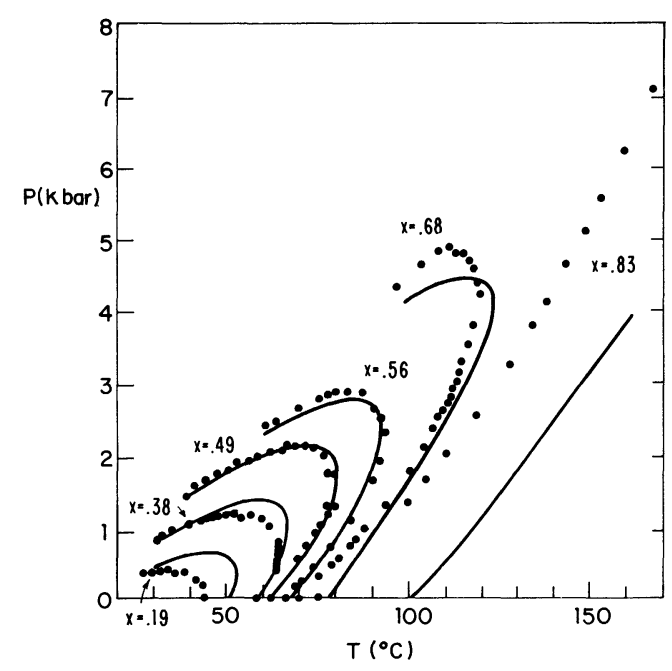

Fig. 2. - Fit of eq. (6) to the reentrant phase diagrams of binary mixtures of CBNA and CBHA, measured by Cladis et al. The concentration $x=$ weight $\mathrm{CBNA} /$ weight [CBHA + CBNA] and the parameters are $T_{1}=42.85{ }^{\circ} \mathrm{C}, T_{2}=43.55^{\circ} \mathrm{C}, T_{3}=25^{\circ} \mathrm{C}$, $t_{1}=38.7{ }^{\circ} \mathrm{C}$ and $\alpha_{\mathrm{N}} / \beta_{\mathrm{N}}=0.0516 \mathrm{kbar} /{ }^{\circ} \mathrm{C}$. The dots and solid lines have the same meaning as for figure 1 .
If we take $P=0$ in eq. (6) (i.e., atmospheric pressure) the nematic-smectic A phase boundary is predicted to have the same form, e.g.,

$$
y-y_{\mathrm{Ns}}+\beta\left(T_{\mathrm{Ns}}-T\right)^{2}=0
$$

that Cladis [2] used to describe her observations on binary mixtures of $p[p$-hexyloxy-benzylidene]-aminobenzonitrile (HBAB) and N-p-cyanobenzylidene-p-noctyloxyaniline (CBOOA). Expressions for $T_{\mathrm{Ns}}$ and $y_{\mathrm{Ns}}$ in terms of the parameters in eq. (6) are easily obtained. Mixtures of 4-cyano-4'-hexyloxybiphenyl (6OCB) and (8OCB) behave similarly [12].

According to the Landau theory there is no essential difference between the high and low temperature nematic phases and experiments support this. For example Cladis's measurements of the bend elastic constant $K_{3}$ in HBAB-CBOOA mixtures behaved similarly on both sides of the smectic phase. Furthermore at concentrations high enough that a smectic phase does not appear (i.e., $x>x_{0}$ ) the standard Landau theory [3] predicts that both the bend and twist elastic constants, $K_{3}$ and $K_{2}$ should diverge as the correlation length. For $x>x_{0}$ this will have the form $\left[\left(T_{\mathrm{Ns}}-T\right)^{2}+(\Delta T)^{2}\right]^{-1 / 2}$. Figure 3 demonstrates the fit between this expression and Cladis's data [2]. Schaetzing et al. [13] have done light scattering studies on the nematic phase of 6OCB-8OCB mixtures as the smectic is approached from both higher and lower temperatures. Although for $x<x_{0}$ the tran-

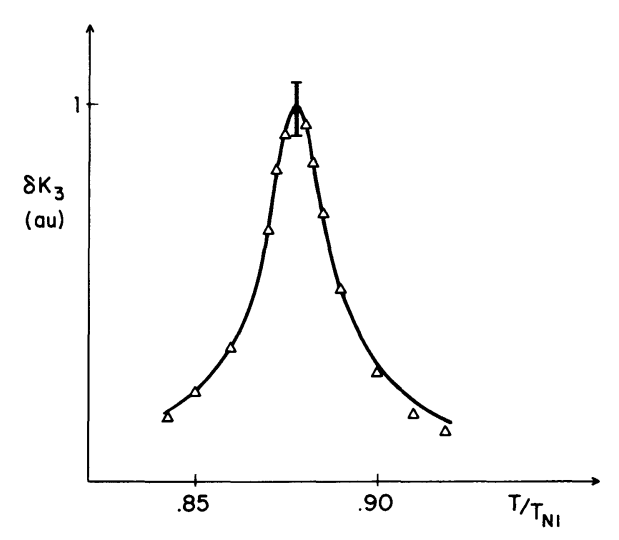

Fig. 3. - Enhancement of the bend elastic constant $K_{3}$ as a function of temperature, in a mixture of $12.67 \%$ by weight of $\mathrm{HBAB}$ in CBOOA, as a function of the reduced temperature $t=T / T_{\mathrm{N} 1}$. The triangles are estimated from the figure 3 of reference [2] by subtracting to the critical field $H_{\mathrm{c}}\left(\propto K_{3}\right)$ at the reduced temperature $T / T_{\mathrm{N} 1}$, the background value $H_{\mathrm{c}}^{0}\left(\propto K_{3}^{0}\right.$, value characteristic of the nematic phase without smectic fluctuations) arbitrarily chosen at $T / T_{\mathrm{N} 1}=0.99$. (The error bar, is an estimate of the inaccuracy resulting from this procedure.) The solid line is calculated from

$$
\delta K_{3} \propto \frac{1}{\sqrt{1+\left(\frac{t-0.878}{0.007}\right)^{2}}}
$$


sitions appear weakly first order and are not well described by a Landau type of mean field theory the critical exponents in both cases are identical (i.e. $v \simeq 0.67 \pm 0.05$ ); again indicating the similarity of the high and low temperature nematic.

In summary we have demonstrated that the principle experimentally observed properties of reentrant nematic phases can be understood simply in terms of an optimum density for smectic ordering. The Landau theory expresses this qualitative idea in quantitative forms through the demonstration that the ratio $\alpha_{N} / \beta_{N}$ required to fit the data is the value expected from independent measurements. Although Clark previously [14] argued that the phase boundary should be elliptical, rather than parabolic, in the $P-T$ plane, the extra terms required to change the parabolic form into the elliptical are small in comparison with the quadratic term in eq. (2).

Acknowledgments. - One of us (PP) would like to thank David Nelson, Mike Stephen, Bob Birgeneau and J. D. Litster for helpful conversations in relation to this manuscript. We would also like to acknowledge ancillary support for this work by the National Science Foundation under Grant No. DMR 76-01111 and the Joint Services Electronics Program under Contract No. N00014-75-C-06-48.

\section{References}

[1] Cladis, P. E., Bogardus, R. K., Daniels, W. B., TayLOR, G. N., Phys. Rev. Lett. 39 (1977) 720.

[2] Cladis, P. E., Phys. Rev. Lett. 35 (1975) 48.

[3] De Gennes, P. G., Solid State Commun. 10 (1972) 753.

[4] MacMillan, W. L., Phys. Rev. A 4 (1971) 1238.

[5] See for example Schlottman, P., J. Low Temp. Phys. 20 (1975) 123.

[6] See for example Anderson, A. C., Reese, W., Wheatley, J. C., Phys. Rev. 130 (1963) 1644.

[7] Landau, L. D. and Lifshitz, E. M., Statistical Physics, Chapters 12 and 14 (Oxford, Pergamon Press) 1958.
[8] For example see Eq. Siggia, E. D., Nelson, D. R., Phys. Rev. B 15 (1977) 1427.

[9] Leadbetter, A., Mol. Cryst. Liq. Cryst. 34 (1977) 231.

[10] Miller, W. H., Dunmur, D. A., 7th Internat. Liq. Cryst. Conf. (Bordeaux, France) 1978 (unpublished).

[11] Liao, Y., Clark, N. A., Pershan, P. S., Phys. Rev. Lett. 30 (1973) 639.

[12] Guillon, D., Cladis, P. E., Stamatoff, J. (to be published).

[13] Schaetzing, R., Davidov, D., Dana, S. S., Litster, J. D., BIRGENEAU, R. J. (to be published).

[14] Clark, N. A., 7th Internat. Liq. Cryst. Conf. (Bordeaux, France), 1978 (unpublished). 